\title{
Voluntades anticipadas al final de la vida. Una aproximación desde la regulación colombiana y en el derecho comparado*
}

\author{
Advanced wishes for the end of life. \\ An approach from the Colombian regulation \\ and in comparative law
}

\section{Vontades antecipadas para o fim da vida. Uma abordagem a partir da regulamentação da Colômbia e no direito comparado}

Fecha de recepción: 3 de agosto de 2015

Fecha de evaluación: 22 de septiembre de 2015

Fecha de aceptación: 26 de octubre de 2015

Disponible en línea: 15 de diciembre de 2015

Piedad Lucía Bolívar Góez**

Ana Isabel Gómez Córdoba***

DOI: http://dx.doi.org/10.18359/rlbi.1444

Cómo citar:

Bolívar Góez, P. L. y Gómez Córdoba, A. I. (2016). Voluntades anticipadas al final de la vida. Una aproximación desde la regulación colombiana y en el derecho comparado. Revista Latinoamericana de Bioética, 16(1), 128-153. DOI: http://dx.doi.org/10.18359/rlbi.1444.

* Artículo de Reflexión.

** Abogada de la Universidad de Medellín, Colombia; especialista en Derecho Comercial, de la Universidad Pontificia Bolivariana, Colombia. En la actualidad, adelanta Maestría en Derechos Humanos, en la Universidad Nacional de Educación a Distancia, Costa Rica. Profesora de las Universidades del Rosario y Jorge Tadeo Lozano, Bogotá, Colombia, en Derecho Médico. Correo electrónico: pbolivar@bolivaryvalenciaasociados.com.

*** Médica Pediatra, especialista en Gerencia en Salud Pública y en Derecho Médico-Sanitario, de la Universidad del Rosario, Bogotá, Colombia; magíster en Bioética, de la Universidad del Bosque, Bogotá, Colombia; doctora en Ciencias Jurídicas, de la Pontificia Universidad Javeriana, Bogotá, Colombia. Vicedecana de la Escuela de Medicina y Ciencias de la Salud, de la Universidad del Rosario y codirectora de la Especialización en Derecho Médico-Sanitario de la misma universidad. Miembro del grupo de investigación en Educación, en la línea Bioética y Derecho Médico Sanitario, de la Universidad del Rosario. Correo electrónico: anai.gomez@urosario.edu.co. 


\section{Resumen}

La relación de los profesionales de la salud con los pacientes tiene como piedra angular el respeto del derecho de las personas a autodeterminarse, a tomar decisiones libres e informadas respecto a su vida, salud y cuerpo. El final de la vida es uno de los más importantes momentos vitales en el cual es definitivo garantizar que el paciente pueda tomar una serie de decisiones sobre múltiples aspectos, como son el deseo o no de ser informado, aceptar o no medidas terapéuticas y de cuidado paliativo, rechazar aquellas que son fútiles, definir quién puede acceder a sus datos clínicos o quién representara su voluntad.; esto con el fin de proteger su derecho a una muerte digna. No obstante, en esta parte de la vida es frecuente que el paciente se encuentre aquejado por patologías que alteran su competencia para decidir, lo cual nos obliga a recurrir a instrumentos que protejan y proyecten su autonomía en el tiempo, como es el caso de las voluntades anticipadas. En Colombia, dos importantes regulaciones han tratado este tema recientemente: la Ley 1733 de 2014 y la Resolución 1216 del 20 de abril del 2015.

El presente artículo pretende, con una perspectiva bioética y jurídica, analizar distintos aspectos de las voluntades anticipadas en Colombia, teniendo como hilo conductor el derecho comparado, de tal forma que permita establecer las condiciones requeridas para garantizar la eficacia y la validez de estas, y determinar los titulares de este derecho y el alcance y la exigibilidad de dichas voluntades.

Palabras clave: voluntad anticipada, muerte digna, eutanasia, consentimiento informado.

\section{Abstract}

The relationship of health professionals with patients is the cornerstone respect for the right of people to self-determination, to make free and informed decisions about their life, health and body. The end of life is one of the most important moments in life which is definitive ensure that the patient can take a number of decisions on many aspects, such as the desire or not to be informed, or not to accept care and therapeutic measures palliative, rejecting those that are futile, define who has access to their clinical data or who represent their will; this in order to protect their right to a dignified death. However, patients are often suffering from diseases which impair their ability to make decisions during this part of their lives, forcing us to resort to instruments to protect their autonomy and project it, as in the case of living wills. Two important regulations have addressed this subject recently in Colombia: Law 1733/ 2014, "regulating palliative care services aimed at the integral management of patients suffering terminal, chronic, degenerative and irreversible diseases," and Resolution $1216,20^{\text {th }}$ April 2015, "leading to compliance with the Constitutional Court's fourth order of ruling T-970/2014 regarding guidelines for the organization and functioning of committees for implementing the right to die with dignity". This article intends to, with a bioethics and legal perspective, analyze the advance wills in Colombia taking the comparative law as a thread, so that it allows establishing the conditions required ensuring the effectiveness and validity of these and determining the holders of this right and the scope and enforceability of such wills.

Keywords: Advance wills, living wills, dignified death, euthanasia, informed consent.

\section{Resumo}

A relação dos profissionais de saúde com os pacientes tem como pedra fundamental o respeito do direito das pessoas à auto-determinarse, para tomar decisões livres e informadas sobre a sua vida, a sua saúde e o seu corpo. O fim da vida é um dos momentos mais importantes vitais no qual é definitivo garantir que o paciente possa tomar uma série de decisões sobre os múltiplos aspectos, tais como são o desejo ou não de ser informado, aceitar ou não as medidas terapêuticas e de cuidado paliativo, rejeitando aquelas que são fúteis, definir quem pode ter acesso aos seus dados clínicos ou quem representara a sua vontade; isto, a fim de proteger o seu direito a uma morte digna. No entanto, nesta parte da vida acontece muitas vezes que o paciente esteja sofrendo muito de patologias que alterem a sua competência para decidir, o qual força-nos a apelar a instrumentos que protejam e projectem a sua independência ao longo do tempo, como é no caso das vontades antecipadas. Na Colômbia, dois regulamentos importantes tem abordado esta questão recentemente: a Lei 1.733 de 2014 e a Resolução 1216 de 20 de abril de 2015.

O presente artigo visa, com uma perspectiva bioética e jurídica, analisar diferentes aspectos das vontades antecipadas na Colômbia, tendo como fio condutor o direito comparado, a fim de estabelecer as condições necessárias para garantir a eficácia e a validade das mesmas, e determinar os detentores desse direito e da abrangência e exigibilidade de tais vontades.

Palavras-chave: vontade antecipada, morte digna, eutanásia, consentimento informado. 


\section{Introducción}

Hasta la década de los sesenta, el papel que las personas desempeñaban en el proceso del cuidado de su propia salud era pasivo; recaía en el profesional de la salud la responsabilidad de tomar decisiones con una perspectiva paternalista, en la cual lo bueno era lo que fuese beneficiente, no maleficente, y acorde con la lex artis de la profesión. Sin embargo, como producto de diferentes factores (entre ellos los movimientos por los derechos de las minorías, los excesos de la autonomía médica y los dilemas relacionados con la interacción de la vida con la tecnología, sobre todo al final de la vida), progresivamente se produjo un cambio en la dinámica de la relación médico-paciente, que obligaba al profesional de la salud a proteger el derecho de las personas a tomar decisiones libres e informadas en relación con su vida, su salud y su integridad corporal; todo ello encontró un amplio desarrollo en la regulación nacional e internacional.

El respeto a la autodeterminación de las personas cobra especial importancia en relación con las decisiones que deben tomarse al final de la vida, y que garantizan el derecho a una muerte digna, como son, entre otras, el deseo de ser informado o no del propio estado de salud; también, poder definir en caso de pérdida de la competencia quién representaría la voluntad del paciente y la autorización para el inicio de medidas de cuidado paliativo, la readecuación terapéutica o la limitación del esfuerzo terapéutico, el rechazo terapéutico, la sedación paliativa y terminal, la terminación anticipada de la vida, el acceso de terceros a los datos personales, la donación de órganos o, incluso, definir el sitio donde se quiere morir, y si se quiere o no contar con asistencia religiosa, entre otras. El derecho a una muerte digna tiene una estrecha relación con el respeto a las creencias, los valores y las necesidades del paciente.

Las decisiones de los pacientes, acordes con su edad y su madurez, se expresan en el proceso de consentimiento informado, el cual es el producto de un proceso de comunicación en el cual se da un acuerdo de voluntades entre el profesional de la salud y el sujeto de cuidado, y el cual tiene unos efectos jurídicos. No obstante, bien sea por la edad del paciente o por una condición que menoscabe su competencia para discernir, no siempre es posible que dicho consentimiento sea otorgado de forma directa, situación en la cual un tercero autorizado ante la ley representa su voluntad. Esta situación se intensifica al final de la vida, como en el caso de enfermedades en fase terminal, crónicas, degenerativas e irreversibles, de alto impacto en la calidad de vida. El consentimiento indirecto, o subrogado, si bien busca proteger el derecho a la autonomía, ofrece limitaciones relacionadas con posibles conflictos de interés del representante, la dificultad de definir el menor interés con la perspectiva del paciente o reconstruir su voluntad hipotética. Un ejemplo de las dificultades que representa no tener una directriz 
previa por parte del paciente en situaciones límite del cuidado al final de la vida es el representado por casos de gran resonancia internacional que han llegado a instancias jurídicas, como son, entre otros, los de Karen Ann Quinlan y de Nancy Cruzan, en Estados Unidos, y el de Eluana Englaro, en Italia.

¿Cómo extender, entonces, el derecho que tienen las personas a autogobernarse y a tomar decisiones sobre su vida y su salud cuando no cuentan con la competencia para hacerlo? Este interrogante es el que nos motivó a hacer una investigación de tipo teórico y descriptiva, sobre las voluntades anticipadas, con las perspectivas ética y jurídica, cuyos resultados expondremos a continuación y los cuales esperamos que sirvan para orientar a los profesionales de la salud, a los pacientes y a los abogados en cuanto a la garantía del derecho a una muerte digna dentro del sistema de salud colombiano. Para cumplir con tal objetivo, haremos una revisión de los más importantes conceptos asociados y de los aspectos presentes en regulaciones foráneas, así como los elementos de existencia y validez jurídica presentes dentro del marco regulatorio colombiano, con una perspectiva crítica.

\section{Definición, carácter y contenidos de la voluntad anticipada}

La voluntad anticipada es la manifestación que hace, de manera unilateral, autónoma y libre, una persona, como sujeto moral, sobre lo que quiere en caso de encontrarse en una determinada condición clínico-patológica que le disminuya o le aniquile su independencia, su autonomía, y en la que no pueda manifestar de manera directa y expresa su consentimiento. Es una proyección en el futuro de las posibles decisiones respecto al ejercicio de sus derechos al final de la vida en relación con su salud y su integridad, y acorde con sus valores, sus creencias y sus necesidades. Tales decisiones están dirigidas a los profesionales de la salud, así como a las personas, los familiares o los allegados que puedan incidir sobre cuestiones al final de la vida del paciente (Howard, 2012, p. 174).

En palabras de Francisco León (2008), "Mediante esta declaración anticipada una persona podrá manifestar su voluntad sobre los cuidados y tratamientos a los que desearía ser sometida en el evento de que se encuentre en una situación en la cual no esté en condiciones de expresar su consentimiento personalmente" (pp. 83-101). Zappalá (2008) define dicha declaración como

[...] aquella declaración o conjunto de declaraciones por medio de las cuales un individuo, dotado de plena capacidad, expresa su voluntad sobre los tratamientos médicos a los que desearía o no ser sometido en la eventualidad de una enfermedad, trauma imprevisto o advertido, vejez o simplemente incapacidad, en consecuencia de los cuales no estaría en condición de expresar su propio consentimiento informado (u oposición al mismo) (p. 24). 
También se define como las "declaraciones unilaterales escritas, dirigidas al personal sanitario y a otras personas significativas, donde una persona mayor de edad expresa los tratamientos que desea o no desea que se le apliquen en diversas circunstancias médicas futuras, para que dirijan la toma de decisiones médicas cuando ella ya no tenga la capacidad de expresarse por sí misma" (Howard, 2012, p. 174).

Las voluntades anticipadas se denominan de múltiples formas: instrucciones previas, directrices previas, plan de cuidado avanzado, directivas médicas anticipadas, declaraciones sobre la propia incapacidad, testamentos vitales o biológicos, entre otras. En el ámbito anglosajón, se conocen como living will.

De este conjunto de denominaciones, la de testamentos vitales o biológicos, entendida como "las declaraciones anticipadas específicamente originadas en caso de enfermedades consideradas extremas o terminales, según las cuales con certeza llevarán al fallecimiento del individuo" (Zappalá, 2008), ha sido la más fuertemente cuestionada, pues los testamentos desde el punto de vista jurídico tienen efecto en el momento de la muerte y regulan, por lo general, cuestiones patrimoniales, además de las requisiciones jurídicas para su validez, en contraste con el objeto de las voluntades anticipadas, las cuales son disposiciones que se hacen para ser ejecutadas en vida (Tobar, 2012, p. 247).
En el ámbito de la salud, living will es la posibilidad que tiene una persona para, de manera libre y espontánea, manifestar su voluntad con el fin de que surta efectos en el futuro, cuando ya no ostentare capacidad para manifestar su voluntad (Leone y Privitera, 1994). Howard (2012) resume así las características de la voluntad anticipada:

- Es un acto jurídico

- Para que produzca su efecto, se requiere que el otorgante se halle en situación de incapacidad de manifestar por sí mismo su voluntad.

- Necesita la solemnidad de que la voluntad se exteriorice por escrito.

- Dicha voluntad debe ser sea inequívoca.

- Es unilateral.

- Es exclusiva de personas físicas.

- Es unipersonal.

- Es personalísima.

- Tiene destinatarios.

- Es revocable.

- Es imprescriptible e incaducable.

- Sus disposiciones son de naturaleza extrapatrimonial.

- Sus efectos se producen en vida de quien la otorga (pp. 175-176).

Son diversos los contenidos u objetos de la voluntad anticipada el cuidado paliativo, la aceptación o el rechazo de tratamientos futuros, la readecuación o la limitación del esfuerzo terapéutico, la designación de una persona que represente al paciente, cuando a este no le 
sea posible expresar su voluntad, para la donación de órganos (con fines médicos, de docencia o de investigación); asimismo, el acceso de información a terceros, el lugar de la muerte, la aceptación o el rechazo de apoyo religioso y la terminación anticipada de la vida. El objeto de las voluntades estará limitado por el marco jurídico vigente en cada país.

\section{Destinatarios, beneficios y problemáticas de las voluntades anticipadas}

Son distintos los destinatarios de las voluntades anticipadas, a saber: el paciente, su familia, los profesionales de la salud y el Estado.

El paciente se beneficia de estas voluntades cuando se le garantiza la posibilidad de elegir de manera anticipada respecto a eventos que pueden afectar su salud, su integridad personal o su vida, y en medio de los cuales las decisiones que se tomen podrán o no proteger su dignidad y sus derechos. En este orden de ideas, es un instrumento que busca proteger la autonomía del paciente, evita la extralimitación terapéutica y favorece el inicio oportuno del cuidado paliativo.

Para los familiares y los allegados, inmersos en el dolor y el sufrimiento que representa una enfermedad en fase terminal o que afecta la calidad de vida, representa un alivio no tener que afrontar decisiones tan difíciles como son la readecuación del esfuerzo terapéutico o la terminación anticipada de la vida, etc.
Para los profesionales de la salud, por su parte, se hace más expedito el cumplimiento de los deberes relacionados con el respeto a la autonomía del paciente y la protección del mejor interés de este, cuando de forma clara hay un direccionamiento sobre el plan de tratamiento. Por otro lado, disminuye el riesgo jurídico, en especial cuando existen discrepancias con los familiares del paciente. Por último, dado que la voluntad anticipada es un documento a través del cual se protegen derechos básicos de los ciudadanos, el Estado es el responsable de su protección y la sociedad en su conjunto debe respetarlo, en desarrollo del principio de solidaridad.

No obstante lo anterior, estos documentos no siempre cumplen con sus propósitos, debido a las "confusiones conceptuales" producto de las múltiples denominaciones, "la falta de preparación de los profesionales y las deficiencias organizativas", el desconocimiento de estas por parte de los pacientes o las falsa creencias de que son un mero trámite que puede ser invalidado por la familia o los médicos tratantes, entre otros factores (Yllera, 2014, p. 88).

Por otra parte, no siempre es posible prever la totalidad de escenarios y de decisiones que pueden presentarse en situaciones agudas, terminales o crónicas e irreversibles. Además, no siempre es clara la redacción del documento de voluntad anticipada, lo cual hace necesario interpretar lo que la persona quería decir. En estos casos, adquiere una especial importancia la existencia de una 
persona designada para hacer cumplir la voluntad del paciente o para decidir en su lugar, acorde con los valores, las creencias y las necesidades del paciente. También son de utilidad las denominadas historias clínicas valóricas, en las cuales el paciente reflexiona sobre distintos aspectos de la vida y de la muerte, y que orientan en la interpretación, o el acceso a cartas o diarios que permitan dilucidar la voluntad interna (Howard, 2012, p. 185). Sin embargo, en caso de persistir dudas, es útil tratar de reconstruir la voluntad hipotética del paciente con la ayuda de familiares y allegados, o acudir a un comité de bioética.

\section{Las voluntades anticipadas en el contexto internacional}

En el contexto internacional, se encuentra un desarrollo jurídico en relación con las voluntades anticipadas.

En Estados Unidos, se acuña en 1969 la expresión "testamento vital" (living will), la cual tenía como objeto permitir que "personas mayores de edad y en pleno juicio de sus facultades mentales, pudieran expresar el deseo de qué hacer con sus vidas en el caso de que su salud llegara a un deterioro grave e irreversible" (Martínez, 2011, p. 10). En 1991, entró en vigencia la Ley de Autodeterminación del Paciente, por la cual se protege el derecho de las personas a rechazar por anticipado un tratamiento y a que la decisión autónoma de los pacientes sea respetada. Contempla un documento en el cual se especifican los tratamientos que el paciente quisiera o no recibir en caso de inconsciencia (living will); un poder escrito por el cual se designa a un tercero para que represente al paciente en el proceso de atención (power attorney durable form); y un documento más extenso en el que en un escenario de final de la vida el paciente estimula su voluntad respecto al cuidado médico (advance directive form).

En el contexto europeo, el convenio para la protección de los derechos humanos y la dignidad del ser humano con respecto a las aplicaciones de la biología y la medicina (Convenio sobre los Derechos Humanos y la Biomedicina), también conocido como Convenio de Oviedo, se refiere a las voluntades anticipadas, respecto a lo cual notifica: "serán tomados en consideración los deseos expresados anteriormente con respecto a una intervención médica por un paciente que, en el momento de la intervención, no se encuentre en situación de expresar su voluntad" (Consejo de Europa, 1997, artículo 9).

La Asamblea Parlamentaria del Consejo de Europa cuenta con la Resolución 613 de 1976 (Consejo de Europa, 1976a), según la cual se considera "que los enfermos terminales quieren morir en paz y con dignidad, con el apoyo y la compañía de familiares y amigos". La Recomendación 779 (Consejo de Europa, 1976b) considera que: "prolongar la vida no debe ser, en sí mismo, el fin exclusivo de la práctica médica, que debe preocuparse igualmente por el alivio del sufrimiento". Y la Recomendación 1418 
(Consejo de Europa, 1999) referente a la "Protección de los derechos humanos y la dignidad de los enfermos terminales y moribundos", con base en el respeto por la autonomía y la autodeterminación del paciente terminal, prescribe la necesidad de adoptar medidas que garanticen una muerte digna cuando el paciente terminal así lo ha decidido de manera libre: libertad que también incluye el derecho a recibir una información veraz y completa, el derecho a no ser informado y el derecho a rechazar alguna propuesta terapéutica.

En España, la Ley 41 de 2002, Básica Reguladora de la Autonomía del Paciente, y con desarrollo normativo posterior en las comunidades autónomas, reguló las voluntades anticipadas ${ }^{1}$. Su articulado incluye su definición, sus requisitos de existencia y su validez jurídica, incluidos el objeto, la forma de exteriorización de la voluntad y la posibilidad de ser revocadas. Se refiere a las voluntades anticipadas como "documento de instrucciones previas" (Jefatura de Estado, Ley 41,2002$)^{2}$, y exige que sean emitidas

1 En la Comunidad Autónoma de Andalucía, el 9 de junio de 2009 fue aprobado el proyecto de Ley de Derechos y Garantías de la Dignidad de las Personas en el Proceso de la Muerte; en Cataluña, desde el 22 de junio de 2002, fecha de la puesta en funcionamiento del Registre Central de Voluntades Anticipadas

2 Ley 41/2002, artículo 11, numerales 1, 2 y 3 : "Por el documento de instrucciones previas, una persona mayor de edad, capaz y libre, manifiesta anticipadamente su voluntad, con objeto de que ésta se cumpla en el momento en que llegue a situaciones en por una persona mayor de edad, capaz y libre. Además, tienen como objeto, por una parte, los cuidados y el tratamiento en salud, frente a circunstancias en las que la persona no se encuentre en capacidad de expresarlos personalmente; por otra, la disposición de "su cuerpo o de los órganos del mismo" (Jefatura de Estado, 2002), cuando ya se ha presentado su deceso. También autorizan la posibilidad de que el paciente delegue en un tercero su interlocución con el equipo de salud para el cumplimiento de su voluntad. Instan, así mismo, a crear procedimientos dentro de los servicios de salud para poder darles cumplimiento. Se especifica también que el objeto de estas no puede ser contrario "al ordenamiento jurídico, a la 'lex artis', ni las que no se correspondan con el supuesto de hecho que el interesado haya previsto en el momento de manifestarlas"; de esta manera se deja "constancia razonada" en la historia clínica del paciente de estas. Si bien estas pueden ser revocadas, debe quedar un registro del hecho en la historia clínica. Adicionalmente, se crea en el Ministerio de Sanidad y Consumo

cuyas circunstancias no sea capaz de expresarlos personalmente, sobre los cuidados y el tratamiento de su salud o, una vez llegado el fallecimiento, sobre el destino de su cuerpo o de los órganos del mismo. El otorgante del documento puede designar, además, un representante para que, llegado el caso, sirva como interlocutor suyo con el médico o el equipo sanitario para procurar el cumplimiento de las instrucciones previas". Ley 18.473 del 3 de abril de 2009, Voluntad anticipada, de Uruguay. Recuperado de http://www.parlamento.gub.uy/leyes/ AccesoTextoLey.asp?Ley=18473\&Anchor= 
el registro nacional de instrucciones previas (Jefatura de Estado, 2007).

En las comunidades autónomas, existen diferencias respecto al requisito de la edad: en Navarra, rige al respecto la Ley Foral 11 de 2002, sobre los mayores de edad y los mayores de 16 años (menores a quienes se les reconoce capacidad); en Andalucía, la Ley 5 de 2003, sobre los menores anticipados, y en Valencia, la Ley 1 de 2003, para menores emancipados (Jiménez, 2009, p. 470).

En Holanda, en 2002 entró en vigencia la Ley de Verificación de la Terminación de la Vida a Petición y Suicidio Asistido, en cuyo artículo 2, apartado 2 se especifica, en lo referente a la eutanasia, que

El médico podrá atender la petición de un paciente, que cuente al menos con dieciséis años de edad, que ya no esté en condiciones de expresar su voluntad pero que estuvo en condiciones de realizar una valoración razonable de sus intereses al respecto antes de pasar a encontrarse en dicho estado de incapacidad y que redactó una declaración por escrito que contenga una petición de terminación de su vida.

Los médicos no serán sancionados si siguen algunas pautas como: 1) la persona tiene que padecer una enfermedad que provoque sufrimientos insoportables sin esperanza alguna de sobrevivir; 2) el enfermo deberá hacer de manera voluntaria y deliberada la petición; 3) deberá intervenir una comisión que juzgará si el médico practicó la eutanasia según las reglas del nuevo marco legal; 4) el médico tiene el deber de comunicar a la comisión la eutanasia practicada por él; 5) en caso de duda, puede acudir al fiscal; 6) las personas de 16 y 17 años podrán decidir independientemente sobre el tema, aunque se tendrá en cuenta la opinión de los padres, mientras que quienes tengan entre 12 y 16 años necesitarán la aprobación de sus progenitores o su tutor (Jiménez, 2009, p. 491).

En Luxemburgo, en 2008 se despenalizó la eutanasia, en el caso de

[...] mayores de edad y menores emancipados, con diagnóstico irreversible y un sufrimiento físico y psíquico constante e insoportable sin perspectivas de mejora y que expongan de manera voluntaria y reiterada, sin expresión externa su deseo de morir. Esta ley considera también la existencia de testamento vital, el cual es un documento donde consta la voluntad del paciente y el cual se archiva en un registro Nacional. (Jiménez, 2009, p. 493).

En Francia, desde 2005 está prohibido el ensañamiento terapéutico ${ }^{3}$, y en 2015
La obstinación, extralimitación o ensaña- miento terapéutico es la recomendación o aplicación por parte del médico de acciones de prevención, diagnóstico, terapéutica o rehabilitación que son inútiles, innecesarias (fútiles), ineficaces o desproporcionadas que prolongan el proceso de muerte. 
la Asamblea Nacional declaró que es una obligación del médico ofrecer a los pacientes la posibilidad de un testamento vital; también admite dentro de esta reglamentación la posibilidad del testamento sustituto y el derecho de los ciudadanos a acceder a una sedación profunda y continuada al final de su recorrido vital; sin embargo, aún no se ha aprobado lo relativo a los cuidados paliativos (Cañas, 17 de marzo de 2015).

En Argentina, la Ley 26.529 consagra en su artículo 11 las directivas anticipadas; señala que

Toda persona capaz, mayor de edad puede disponer directivas anticipadas sobre su salud, pudiendo consentir o rechazar determinados tratamientos médicos, preventivos o paliativos, y decisiones relativas a su salud. Las directivas deberán ser aceptadas por el médico a cargo, salvo las que impliquen desarrollar prácticas eutanásicas, las que se tendrán como inexistentes. (Senado y Cámara de Diputados de la Nación Argentina, 2009).

Esta norma fue posteriormente adicionada definiendo requisitos formales para su otorgamiento.

En Uruguay, la normatividad de ese país en la Ley 18.473, sobre voluntad anticipada, exige que la manifestación de voluntad conste documentalmente y que la persona que la otorga sea mayor de edad y con aptitud psíquica para decidir oponerse a la aplicación de tratamientos médicos, salvo si con ello puede afectar a terceros; concede, además, la posibilidad de una voluntad anticipada de oposición a tratamientos, pero no los cuidados paliativos, en caso de encontrarse ante una patología terminal, incurable e irreversible. Consagra la ley de Uruguay el concepto de la voluntad diferida, en caso de que el paciente no lo pueda hacer; pero si concurren varias de las personas, exige unanimidad en su decisión. Finalmente, consagra la posibilidad de que el paciente se retracte de su manifestación previa en torno a tratamientos y terapéuticas (Senado y Cámara de representantes de la República Oriental de Uruguay, 2009).

En México, desde 2008 se consagró legalmente la posibilidad de expedir voluntades anticipadas a personas mayores de edad, y en 2011 se aligeraron sus requisitos para hacerla más asequible a los pacientes y al mismo tiempo exigiendo a los familiares el respeto por la decisión de este, pero dotándolos de facultades para exigir al cuerpo sanitario la observancia de la voluntad del paciente.

En términos generales, los Estados Unidos Mexicanos han implementado una regulación que propende a proteger la dignidad de los pacientes prohibiendo el acortamiento de la vida, limitando la voluntad únicamente a las decisiones de rechazo de posibilidades terapéuticas y a recibir cuidados paliativos (Asamblea 
Legislativa del Distrito Federal, s. f.). Los Estados Unidos Mexicanos prohíben $^{4}$ que tengan como objeto el acortamiento intencional de la vida (Cantú et al., 2012). La voluntad anticipada puede presentarse ante notario, ante un profesional de la salud de institución privada o pública, o representarla, cuando el paciente esté impedido para hacerlo, por un familiar o una persona legalmente responsable en orden de prelación. El médico deberá justificar el diagnóstico y presentarlo ante el comité de ética hospitalaria, para determinar si, en efecto, el paciente está en una etapa terminal. Establece además unos requisitos para revocarla, como: 1) que no sea libre, 2) que se busque un beneficio del suscriptor, 3 ) cuando la voluntad no sea inequívoca, y 4) cuando así lo decida el paciente (Ariza et al., 2008).

A manera de resumen, en el contexto internacional, a pesar de las múltiples denominaciones existentes, las voluntades anticipadas son un instrumento de protección del derecho a la autodeterminación de los pacientes. Las regulaciones de los distintos países coinciden en los siguientes aspectos: 1) solo las personas capaces legalmente y en pleno uso de sus facultades mentales pueden suscribirlas; 2) la exteriorización de los deseos del paciente debe ser expresa y por

\footnotetext{
$4 \quad$ "Esta norma no permite ni faculta, bajo circunstancia alguna, la realización de conductas que tengan como consecuencia el acortamiento intencional de la vida" (Cantú et al., 2012, p. 13).
}

escrito, y 4) la voluntad debe ser libre, informada, inequívoca, seria y reiterada. Son diversos los objetos jurídicos de la voluntad anticipada y dependerán del grado de desarrollo jurídico respecto a temáticas al final de la vida, como el cuidado paliativo, la sedación terminal o la eutanasia; además, incluyen en general la readecuación terapéutica, el rechazo terapéutico, la donación, el uso de órganos con fines de investigación, la designación de un sustituto para tomar decisiones o para corroborar el cumplimiento de la propia voluntad y su aplicación en casos de enfermedad terminal o crónica avanzada.

Como elementos diferenciales, algunos países tienen requisitos de solemnidad, como su certificación ante notario y con testigos, y la existencia de registros nacionales que favorezcan su aplicación expedita.

\section{Las voluntades anticipadas en Colombia}

En Colombia el derecho a una muerte digna -temática en la que se contextualizan las voluntades anticipadas- tiene importantes antecedentes jurídicos en los derechos humanos, la Constitución Política de Colombia, la Ley 23 de 1981, la Resolución 13437 de 1991, el Decreto 2493 de 2004, el Código Penal Colombiano, la Sentencia C-239/97, la Ley 1737 de 2014, la Sentencia T-940 de 2014, y la Resolución 1216 del 20 de abril de 2015. 
La Ley 23 de 1981, que aborda los aspectos deontológicos de la profesión médica, prescribe en su artículo 13: "el médico usará los métodos y medicamentos a su disposición o alcance, mientras subsista la esperanza de aliviar o curar la enfermedad. Cuando exista diagnóstico de muerte cerebral, no es su obligación mantener el funcionamiento de otros órganos o aparatos por medios artificiales" (Congreso de Colombia, 1981, artículo 13).

La Resolución 13437 de 1991, por la cual se constituyen los comités de ética hospitalaria y se adopta el Decálogo de los Derechos de los Pacientes, consagra el derecho de las personas a morir con dignidad y a que se les respete su voluntad de permitir que el proceso de la muerte siga su curso natural en la fase terminal de su enfermedad, así como los derechos a aceptar o a rehusar la asistencia espiritual y a donar órganos o tejidos (Ministerio de Salud, 1991).

El Decreto 2493 de 2004, por el cual se reglamentan parcialmente las Leyes 9a de 1979 y 73 de 1988, en relación con los componentes anatómicos, aporta en su artículo 2 la definición de muerte encefálica, así como la voluntad de donación después de la muerte. El artículo 17 señala en su Parágrafo $1^{\circ}$.

La voluntad manifestada por la persona donante en la forma señalada en el presente artículo, prevalecerá sobre la de sus deudos. El donante podrá revocar en cualquier tiempo, en forma total o parcial, antes de la ablación, la donación de órganos o componentes anatómicos, con el mismo procedimiento que utilizó para la manifestación de donación. (Presidencia de la República de Colombia, 2004, artículos 2 y 17).

En este orden de ideas, se puede afirmar que el primer objeto de las voluntades anticipadas en Colombia fue la donación de órganos. El Código Penal Colombiano tipifica como delitos el homicidio por piedad y el suicidio asistido.

La Sentencia C-239/97, del magistrado Carlos Gaviria, se refiere a la temática del homicidio por piedad, e indica que cuando una persona capaz legalmente, aquejada de una enfermedad terminal y con sufrimiento y dolor que desde su perspectiva hace indigna su vida, solicite de manera libre, informada y reiterada a su médico que finalice con su vida, si este lo hiciere motivado por la piedad, dicha acción no acarreará la pena establecida por el Código Penal para el homicidio por piedad. En tal caso, la manifestación de la voluntad se hace mediante consentimiento directo.

Más recientemente, y después de un tortuoso proceso de oposiciones y de objeciones, se logró que se expidiera la Ley 1733 de 2014, la cual regula los servicios de cuidados paliativos y "reglamenta el derecho que tienen las personas con enfermedades en fase terminal, crónicas, degenerativas e irreversibles, a la atención en cuidados paliativos que pretende mejorar la calidad de vida, tanto de los pacientes que 
afrontan estas enfermedades, como de sus familias, mediante un tratamiento integral del dolor, el alivio del sufrimiento y otros síntomas, teniendo en cuenta sus aspectos psicopatológicos, físicos, emocionales, sociales y espirituales, de acuerdo con las guías de práctica clínica que establezca el Ministerio de Salud y Protección Social para cada patología" (Congreso de Colombia, 2014, artículo 1)5. En

5 Se entiende por enfermo en fase terminal "a todo aquel que es portador de una enfermedad o condición patológica grave, que haya sido diagnosticada en forma precisa por un médico experto, que demuestre un carácter progresivo e irreversible, con pronóstico fatal próximo o en plazo relativamente breve, que no sea susceptible de un tratamiento curativo y de eficacia comprobada, que permita modificar el pronóstico de muerte próxima; o cuando los recursos terapéuticos utilizados con fines curativos han dejado de ser eficaces". Y "Se define como enfermedad crónica, degenerativa e irreversible de alto impacto en la calidad de vida aquella que es de larga duración, que ocasione grave pérdida de la calidad de vida, que demuestre un carácter progresivo e irreversible que impida esperar su resolución definitiva o curación y que haya sido diagnosticada en forma adecuada por un médico experto". Se entiende por cuidado paliativo "Son los cuidados apropiados para el paciente con una enfermedad terminal, crónica, degenerativa e irreversible donde el control del dolor y otros síntomas, requieren, además del apoyo médico, social y espiritual, de apoyo psicológico y familiar, durante la enfermedad y el duelo. El objetivo de los cuidados paliativos es lograr la mejor calidad de vida posible para el paciente y su familia. La medicina paliativa afirma la vida y considera el morir como un proceso normal. Parágrafo. El médico usará los métodos y medicamentos a su disposición esta ley se exponen, en el artículo 5, los derechos de los pacientes al final de sus vidas, incluido el derecho a subscribir el documento de voluntad anticipada, incluyendo el derecho al cuidado paliativo y a la toma de decisiones sobre este tipo de atención; a la información; a una segunda opinión; a participar de forma activa en el proceso de atención, y los derechos de los niños y adolescentes y de los familiares. El artículo 5.4 se consagra al derecho a subscribir voluntades anticipadas como

Toda persona capaz, sana o en estado de enfermedad, en pleno uso de sus facultades legales y mentales, con total conocimiento de las implicaciones que acarrea el presente derecho podrá suscribir el documento de Voluntad Anticipada. En este, quien lo suscriba indicará sus decisiones, en el caso de estar atravesando una enfermedad terminal, crónica, degenerativa e irreversible de alto impacto en la calidad de vida de no someterse a tratamientos médicos innecesarios que eviten prolongar una vida digna en el paciente y en el caso de muerte su disposición o no de donar órganos. (Congreso de Colombia, 2014, artículo 5).

o alcance, mientras subsista la esperanza de aliviar o curar la enfermedad. Cuando exista diagnóstico de muerte cerebral, no es su obligación mantener el funcionamiento de otros órganos o aparatos por medios artificiales, siempre y cuando el paciente no sea apto para donar órganos" (artículos $2,3$ y 4$)$. 
Para resumir, la Ley 1733 de 2014 establece los siguientes elementos de existencia y validez jurídica de las voluntades anticipadas: 1) deben provenir de una persona mayor de 18 años, competente, que puede encontrarse sana o aquejada de una enfermedad terminal o crónica; 2) debe estar precedida de información, la cual incluye los derechos que tienen las personas al final de la vida; 3) su objeto debe ser lícito, incluido el rechazo a tratamientos fútiles y a la donación de órganos, y 4) la exteriorización de la voluntad debe ser expresa y por escrito. Lamentablemente, no se especifican en esta ley otros objetos lícitos de las voluntades anticipadas, las cuales hacen parte de los derechos de los pacientes, como: 1) la autorización para el acceso a datos clínicos de carácter personal; 2) la designación de un representante; 3) el deseo de morir en casa y en compañía de quienes se quiere estar rodeado al final; 4) si se desea o no asistencia religiosa o espiritual, o la donación de órganos con fines de investigación y docencia. Tampoco se contemplan otros medios para exteriorizar de manera inequívoca la voluntad, como videos, grabaciones u otros medios electrónicos.

La Sentencia T-940 de 2014, del magistrado Luis Ernesto Vargas Silva, retoma la necesidad de reglamentar la muerte anticipada y ordena al Ministerio de Salud "que emita una directriz y disponga todo lo necesario para que los Hospitales, Clínicas, IPS, EPS y, en general, prestadores del servicio de salud, conformen el comité interdisciplinario", que deberán estudiar las solicitudes de muerte anticipada para verificar que se cumplan los criterios de seguridad jurídica para los profesionales de la salud que acojan las solicitudes de muerte anticipada de pacientes en fase terminal de sus enfermedades y garanticen la oportunidad en la protección de sus derechos. También indica que el "Ministerio deberá sugerir a los médicos un protocolo médico que será discutido por expertos de distintas disciplinas y que servirá como referente para los procedimientos tendientes a garantizar el derecho a morir dignamente". La fundamentación de proteger el derecho a una muerte digna se inspira en la convicción de que "condenar a una persona a prolongar por un tiempo escaso su existencia, cuando no lo desea y padece profundas aflicciones, equivale no sólo a un trato cruel e inhumano, prohibido por la Carta (CP art.12), sino a una anulación de su dignidad y de su autonomía como sujeto moral".

Los siguientes son elementos que excluyen el carácter delictivo de la acción de propiciar la muerte anticipada de un paciente:

(i) debe mediar el consentimiento del sujeto pasivo. Pero ese consentimiento debe ser libre e informado, lo cual significa que debe ser manifestado por una persona "con capacidad de comprender la situación en que se encuentra". Es decir, el consentimiento implica que el paciente posee información seria, fiable y precisa, pero además cuenta con capacidad 
intelectual suficiente para tomar la decisión. Para garantizar ese consentimiento (EDAD); (ii) el sujeto activo debe ser un médico pues es él el único capaz de brindarle la información precisa al paciente, pero además las condiciones para morir dignamente. En caso de que no sea un médico, el consentimiento estará viciado y por tanto, habrá delito; (iii) el paciente debe padecer una enfermedad terminal que le cause sufrimiento, pues sin ello el elemento subjetivo de la piedad desaparecería. (Colombia, Ministerio de Salud y Protección Social, 2015).

Acorde con la solicitud de la Corte a ese respecto, el Ministerio de Salud y Protección Social expide la Resolución 1216 de 2015. En esta se afirma que "el derecho fundamental a vivir en forma digna implica el derecho fundamental a morir con dignidad", y tiene como objeto impartir las "directrices para la conformación y funcionamiento de los comités Científico-disciplinarios para el derecho a morir con dignidad", los cuales actúan en los casos y bajo las condiciones establecidas en Sentencias C-239 de 1997 y T-970 de 2014. Establece como criterio de garantía para el derecho fundamental a morir con dignidad "la prevalencia de la autonomía del paciente, la celeridad, la oportunidad e imparcialidad".

En el artículo 15 de la Resolución 1216 de 2015 se explican las características del consentimiento para la muerte an- ticipada y se incluye la posibilidad de voluntad anticipada o testamento vital para tal fin. Se afirma, en ese sentido, lo siguiente:

Podrá solicitar procedimiento de morir con dignidad ante su médico tratante quien valorara la condición de enfermedad terminal. El consentimiento debe ser expresado de manera libre informada e inequívoca para que se aplique el procedimiento para garantizar su derecho a morir con dignidad. El consentimiento puede ser previo a la enfermedad terminal, cuando el paciente haya manifestado antes de la misma, su voluntad en tal sentido. Los documentos de voluntades anticipadas o testamentos vitales, para el caso en particular, se consideran manifestaciones validas de consentimiento y deberán ser respetadas como tales. En caso de que la persona mayor de edad se encuentre en incapacidad legal o bajo la existencia de circunstancias que le impidan manifestar su voluntad, dicha solicitud podrá ser presentada por quienes estén legitimados para dar el consentimiento sustituto, siempre y cuando la voluntad del paciente haya sido expresada previamente mediante un documento de voluntad anticipada o testamento vital y requiriéndose por parte de los familiares, que igualmente se deje constancia escrita de tal voluntad. (Ministerio de Salud y Protección Social, 2015). 
La voluntad anticipada para muerte anticipada solo será aplicable en el caso de enfermedades en fase terminal, y se acoge para estos fines la definición que brinda la Ley 1733 de 2014, respecto a cuidados paliativos. Sobre el particular, queda establecido que:

Se define como enfermo en fase terminal a todo aquel que es portador de una enfermedad o condición patológica grave, que haya sido diagnosticada en forma precisa por un médico experto, que demuestre un carácter progresivo e irreversible, con pronóstico fatal próximo o en plazo relativamente breve, que no sea susceptible de un tratamiento curativo y de eficacia comprobada, que permita modificar el pronóstico de muerte próxima; o cuando los recursos terapéuticos utilizados con fines curativos han dejado de ser eficaces.

Se especifica en el artículo 2 (parágrafo) que ante controversia en este aspecto se podrá recurrir a segunda opinión o a la opinión de un grupo de expertos. Así mismo, se protege la libertad del paciente a desistir de la solicitud de morir con dignidad, pues en el artículo 17 se especifica: "En cualquier momento del proceso el paciente o, en caso de consentimiento sustituto, quienes estén legitimados para tomar la decisión, podrán desistir de la misma y optar por otras alternativas". En nuestra opinión, esta es una interpretación desafortunada, debido a que desistir del consentimiento sustituto no aplica para la terminación anticipada de la vida en el caso de una voluntad anticipada; realmente, se trata de una representación de la voluntad y no de una sustitución. Esto solo sería posible en caso de que el paciente hubiese cambiado su voluntad anticipada.

En Colombia, la regulación no contempla la existencia de sistemas informativos o registros nacionales de voluntades anticipadas que sean de fácil consulta por parte de los profesionales de la salud para orientar la toma de decisiones. Esta función ha sido realizada de forma privada por la Fundación Pro Derecho a Morir Dignamente (s. f.), la cual "promueve el respeto al derecho que tiene toda persona de expresar su voluntad anticipada sobre lo que considera muerte digna, de acuerdo con sus planes de vida, calidad de la misma y dignidad"; especialmente, en el caso de "enfermos con expectativa de vida reducida debido a variables de edad o importante compromiso de salud y calidad de vida", e incluye el hecho de poder decidir sobre atención intrahospitalaria, cuidados Paliativos (contexto hospitalario o domiciliario), doble efecto, sedación terminal y muerte médicamente asistida" (s. p.).

No obstante lo anterior, y pese a contar con un desarrollo jurídico en el país, existen barreras para el adecuado uso de las voluntades anticipadas, como por ejemplo: 1) la renuencia de las personas a reflexionar sobre su propia muerte, 2) el 
hecho de que las voluntades anticipadas no se encuentran muy difundidas, 3) la inexistencia de sistemas de información asociados al sistema de salud que puedan ser consultados por los profesionales de la salud, 4) la confusión entre los diferentes objetos de las voluntades (en especial, entre rechazo y readecuación terapéutica y eutanasia), 5) la falta de claridad respecto a procedimientos para su puesta en práctica, y 6) la terminología que puede dar lugar a dificultades en la interpretación.

\section{Validez de la voluntad anticipada en Colombia}

Dado que la voluntad anticipada es un acto jurídico, deben entonces ser observadas las condiciones formales que la ley exige para su reconocimiento, de tal forma que se produzcan los efectos jurídicos expresados por quien, de forma anticipada, ha indicado su voluntad respecto al cuidado de su salud para que sea cumplida en el futuro.

En términos generales, para que dicha voluntad exista y sea válida, debe:

- Estar precedida de la información requerida para la toma de decisión.

- Provenir del paciente o de quien lo represente ante la ley.

- Ser emitida por una persona capaz legalmente.

- Ser emitida por una persona competente.

- Tener objeto y causa lícita.
- Ser exteriorizada.

- Estar exenta de vicios.

- Ser seria.

- Cumplir con las formalidades requeridas.

\section{Información}

Los derechos de las personas al final de la vida, enumerados la Resolución 13437 de 1991, en la Ley 1737 de 2014 y en la Resolución 1216 del 20 de abril del 2015, determinan los contenidos de la información que debe preceder la exteriorización de la voluntad anticipada.

En este orden de ideas, los destinatarios de este tipo de información no deberían ser únicamente personas que se encuentren aquejadas de enfermedades en fase terminal o crónicas, degenerativas o irreversibles, sino toda persona que pueda ejercer el derecho a expresar una voluntad anticipada. Ninguna persona se halla exenta de sufrir una situación aguda que le impida ejercer su derecho a autodeterminarse.

En el proceso de información, el médico debe explicar el derecho a suscribir una voluntad anticipada, así como los posibles objetos de esta, la posibilidad de que dicha voluntad sea revocada en el tiempo y la garantía de que esta no será modificada por la familia ni por profesionales de la salud cuando el paciente ya no pueda decidir. 


\section{Capacidad y competencia}

La Ley 1733 de 2014 se refiere a estos requisitos, la capacidad y la competencia como: "Toda persona capaz, sana o en estado de enfermedad, en pleno uso de sus facultades legales y mentales". Esta descripción parece establecer una diferencia entre la capacidad legal y la competencia, o capacidad para discernir. Otro tanto sucede con la Resolución 1216 de 2015, en relación con la voluntad anticipada para muerte anticipada.

En general se entiende la capacidad como un concepto jurídico, referente a la aptitud que tienen los pacientes para ejercer sus derechos. Se es capaz legalmente cuando se alcanza la mayoría de edad, que en Colombia son los 18 años. La competencia es la aptitud para realizar determinada acción. Es un concepto técnico y se refiere más a la evaluación de las habilidades psicológicas y cognoscitivas de los pacientes (Ortiz, 2009, pp. 18-21).

Una persona capaz es la que está facultada para autodeterminarse y definir su ruta de vida en pro de sus propios objetivos, teniendo como límite el derecho de los demás. Al referirse a la autonomía personal, Beauchamp y Childress consideran que una persona autónoma es la que actúa intencionalmente, con conocimiento suficiente y sin influencias que determinen su acción ${ }^{7}$, facultad que para su ejercicio pleno requiere la ilustración previa, completa, adecuada y suficiente de la persona, para tomar una decisión libre de error (2009, p. 101).

En este orden de ideas, en Colombia únicamente los mayores de 18 años en pleno uso de sus facultades mentales pueden suscribir voluntades anticipadas. Sin embargo, los abordajes hechos en la Ley 1733 de 2014 y en la Resolución 1216 de 2015 son contrarios a diversos pronunciamientos internacionales y nacionales en los que se ha reconocido el derecho de los menores de edad a tomar decisiones en distintas esferas de su vida, incluyendo su propia salud y su integridad corporal. Incluso, la Ley de Cuidados Paliativos es contradictoria, pues reconoce el derecho de los niños y los adolescentes, y posibilita que estos últimos (entre los 14 y los 18 años) sean consultados al momento de decidir sobre los cuidados paliativos ${ }^{6}$.

Pero, iel derecho a suscribir una voluntad anticipada se debe reducir únicamente a las personas que sean consideradas legalmente capaces, de conformidad con la ley (es decir, que sean mayores de edad y tengan plena capacidad para ejercer actos jurídicos)? En nuestra opinión, este es un hito temporal que dista del reconocimiento del derecho a la dignidad, a la autodeterminación, a la libertad, a la intimidad de las personas.

6 Se refiere a los derechos a ser o no informado, a participar en la toma de decisiones de limitación del esfuerzo terapéutico y de cuidado paliativo, así como el derecho a la intimidad. 
A continuación se expondrán algunos antecedentes en los cuales se fundamenta el derecho de los menores de edad a decidir sobre su vida, su salud y su integridad.

Históricamente, el menor de edad era sustraído de las exigencias y las consideraciones del Estado de derecho; no había consideración alguna por la protección de sus derechos de manera integral. Posteriormente, se consideró que los menores son objeto de derechos y por ello se desarrollaron unas políticas públicas que condujeron a protegerlo en contra del abandono físico y moral, pero con un sentido paternalista dentro del cual el padre para proteger a sus hijos tenía derechos sobre él; no era el derecho del menor a ser protegido, sino el derecho del padre a decidir los designios de su hijo. Con la Declaración Universal de los Derechos del Niño, se reconocen plenamente los derechos de los menores y de los adolescentes, y es un deber de la patria potestad dirigir la formación de sus hijos al máximo desarrollo de su autonomía ${ }^{7}$.

\footnotetext{
Aprobada por la Asamblea General de las Naciones Unidas en noviembre de 1989 , con cuatro instrumentos: la Convención Internacional de los Derechos del Niño; las Reglas Mínimas de las Naciones Unidas para la Administración de la Justicia Juvenil, o Reglas de Beijing; las Reglas Mínimas de las Naciones Unidas para la Protección de los Jóvenes Privados de Libertad, y las Directrices de las Naciones Unidas para la Prevención de la Delincuencia Juvenil -Directrices de Riad-.
}

La Declaración de Ottawa, de la Asociación Médica Mundial, sobre el derecho del niño a la atención médica, exige considerar los derechos de los niños al tomar decisiones sanitarias; en relación con el niño maduro según el criterio médico, el cual no puede ser otro que el interés superior, este tendrá derecho a tomar sus propias decisiones (Asociación Médica Mundial, 1998).

La Convención sobre los Derechos del Niño reitera la obligación del Estado de proteger el consentimiento del menor, para lo cual dispone:

"Los Estados partes garantizarán al niño que éste en condiciones de formarse un juicio propio el derecho de expresar su opinión libremente en todos los asuntos que afectan al niño, teniéndose en cuentas las opiniones del niño, en función de la edad y madurez del niño" (Unicef, 1989, artículo 12.1).

Es decir, exige que el Estado mismo vele con celo por la opinión de los niños en todos aspectos que lo afecten; en primer lugar se hallan los que afectan el derecho a la vida, a la integridad personal, a su desarrollo en lo que tiene que ver con el análisis que nos ocupa.

En Colombia, la Constitución Política de Colombia (CPC) de 19910 estipula una protección especial a los niños señalando expresamente la protección de su derecho a la libre expresión de su opinión, lo cual permite concluir que 
en materia sanitaria su voluntad debe ser tenida en cuenta: así,

"son derechos fundamentales de los mismos la vida, la integridad física, la salud y la seguridad social, la alimentación equilibrada, su nombre y nacionalidad, tener una familia y no ser separados de ella, el cuidado y amor, la educación y la cultura, la recreación y la libre expresión de su opinión" (artículo 44).

Pero también la Ley 906 de 2004, en su artículo 383, considera hábil al menor de 12 años para rendir testimonio sin reservas en su valoración, con las únicas exigencias de que este no puede ser juramentado y de que deberá ser acompañado de su representante legal o de un pariente mayor de edad. Y en materia penal, es la misma ley la fuente para aceptar que se reconoce en determinadas circunstancias capacidad al menor de edad frente a ciertos actos, hasta llegar incluso a ser sujetos de imputación penal. Se parte de que será imputable la persona a quien pueden atribuirse las consecuencias de los actos violatorios de conductas que describen delitos, infracciones o contravenciones, de tal manera que, según el Código de la Infancia y la Adolescencia (Ley 1098 de 2008), los niños y los adolescentes son reconocidos como sujetos de derechos.

Por otra parte, también se encuentran numerosos antecedentes sobre el ejercicio de la autonomía de los menores de edad en la jurisprudencia colombiana, y en los cuales se reconoce el derecho de los mayores de cinco años de edad a participar en la toma de decisiones dentro del marco de la atención en salud que puedan llegar a afectar su proyecto de vida. Se pondera el principio de autonomía frente al de beneficencia, en razón de la urgencia del tratamiento, la afectación de la autonomía actual y futura, el carácter ordinario o invasivo del procedimiento y la edad del menor ${ }^{8}$.

Lo anterior conduce a concluir que no se plantea una edad unificada para otorgar validez a los actos de los menores de edad, quienes son titulares de derechos, de tal manera que la autonomía de la voluntad no puede verse restringida por la capacidad de ejercicio.

Sin embargo, a pesar de mencionarse en el objeto de la Ley 1733 que esta "reglamenta el derecho que tienen las personas", en su artículo 5 consagra los derechos de los pacientes, y en el numeral 5 reconoce el derecho a suscribir la voluntad anticipada "a toda persona capaz"; expresión que, con el fin de no dejar de lado los avances jurisprudenciales en la protección de la autonomía de los menores de edad, se refiere a la aptitud para adoptar decisiones de manera libre, espontánea y persistente, y no a la mayoría de edad.

8 Son numerosos los pronunciamientos de la Corte en relación con la participación de los menores de edad y los límites al consentimiento otorgado por los padres en los casos de reasignación de género o en estados intersexuales. Es el caso de las Sentencias T-477 de 1995, SU-337 de 1999, y T-551 de 1999. 


\section{Causa y objeto lícito}

La causa lícita se define como lo que da origen al acto, a la voluntad anticipada, y no es otra cosa sino el ejercicio de los derechos del paciente, que no puede contrariar la ley, el orden público ni las buenas costumbres. Para Pothier, dicha causa busca la protección de la moral (citado por Turcott, s. f., p. 111); además, debe ser real, y de allí que se exija que la condición clínica del paciente lo defina como en un estadio terminal de su enfermedad, sin posibilidades de recuperación y que le cause un gran sufrimiento, condición que deberá ser confirmada por un médico.

El objeto lícito será el fin de esta manifestación de voluntad del paciente, de quien lo represente o de la persona en quien el paciente hubiere confiado su decisión. Es un derecho que se les reconoce a los pacientes y que representa una dimensión del respeto a la autonomía de estos.

La voluntad anticipada es un acto humano con una finalidad determinada: el ejercicio de su autonomía en protección de la autodeterminación y de la dignidad, finalidad que en ese caso es consciente y voluntaria, a través de la cual el paciente opta por una buena muerte sin dolor o sufrimiento físico o psicológico, la que es digna con la perspectiva de las creencias, los valores y las necesidades del paciente, sin que su vida se prolongue de manera innecesaria.
El objeto de las voluntades anticipadas es adoptar decisiones que deben ser observadas en el momento en el cual no pueda discernir, cuando no tenga conciencia o cuando no se halle en condiciones de manifestar su voluntad. Estas voluntades pueden estar dirigidas a:

- Evitar que le sean administrados tratamientos fútiles; es decir: inútiles, peligrosos, ineficaces, desproporcionados; que no brindan ningún beneficio clínico al paciente; que no mejoran el pronóstico, los síntomas ni las enfermedades intercurrentes, y que solo producen sufrimiento, angustia y dolor. Rechazar cualquier esfuerzo terapéutico que pretenda el mantenimiento vital sin posibilidades de recuperación y que, por el contrario, prolongan de manera innecesaria el proceso de morir.

- Decidir ser donante de órganos.

- Permitir el acceso a los datos clínicos a un tercero, dado que en ellos se encuentra información salvaguardada por el derecho a la protección de datos personales.

- Designar a un representante, a una persona que o bien sustituya o bien subrogue la voluntad del paciente. Esto significa que se haga cumplir al pie de la letra la voluntad exteriorizada previamente a quien pueda tomar las decisiones que deban ser adoptadas al final de la vida, acorde con los valores y las creencias del paciente. 
- Manifestar el deseo de no permanecer en un centro hospitalario, sino en su casa y rodeado de sus seres queridos al final de su vida.

- Solicitar o no el apoyo espiritual, religioso y moral que comulga con sus creencias y sus necesidades.

Si bien característicamente se trata de un acto jurídico de carácter unilateral, en el caso de la designación de un representante para tomar decisiones cuando la persona esté en situación de incapacidad, se requerirá su aceptación; es decir, un acuerdo de voluntades (Howard, 2012, p. 176).

\section{Solemnidades}

No hay duda de cuán necesario es documentar la manifestación de la propia voluntad, en la que puedan verificarse las condiciones de su existencia y su validez; sin embargo, en Colombia la Ley 1733, mediante la cual se regulan los servicios de cuidados paliativos para el manejo integral de los pacientes con enfermedades terminales, establece el mencionado derecho en el numeral 4 de su artículo 5, al consagrar que:

Toda persona capaz, sana o en estado de enfermedad, en pleno uso de sus facultades legales y mentales, con total conocimiento de las implicaciones que acarrea el presente derecho podrá suscribir el documento de Voluntad Anticipada. En este, quien lo suscriba indicará sus decisiones, en el caso de estar atravesando una enfermedad terminal, crónica, degenerativa e irreversible de alto impacto en la calidad formada, inequívoca, pero formal o informal.

\section{Conclusiones}

Las voluntades anticipadas son instrumentos útiles para proyectar la protección del derecho a la autodeterminación de las personas, especialmente en situaciones en las cuales no son competentes para hacer juicios razonados. Se trata de un documento en el que una persona plasma su decisión, adoptada de manera libre, ilustrada y autónoma, frente a una situación de salud en la que no sean predicables su aptitud ni su capacidad para discernir sobre conductas médicas, o en incapacidad física, de abstención terapéutica o de garantía de no ser expuesta a sufrimientos innecesarios y fútiles o de ponerle fin a su propia existencia.

Este tipo de documentos tienen su soporte en un amplio desarrollo regulatorio nacional e internacional; no obstante, de un país a otro hay diferencias en cuanto a la forma de denominarlas, a los pacientes a quienes van dirigidas, al objeto de estos, a los procedimientos y a los formatos para su implementación, a los requisitos de solemnidad de este tipo de documentos, a la existencia o no de registros nacionales de consulta y a la obligatoriedad o no por parte de los profesionales de informar a los pacientes sobre la posibilidad de tener una voluntad anticipada. 
En Colombia, la inclusión de la voluntad anticipada explícita dentro del marco regulatorio colombiano es reciente (por ejemplo, en la Ley de Cuidados Paliativos); sin embargo, históricamente este tipo de documentos habían sido utilizados por los pacientes, sobre todo con el respaldo de la Fundación Pro Derecho a Morir Dignamente.

En Colombia, hay un amplio rango de objeto para las voluntades anticipadas: entre otros, la designación de un tercero decisor, la aceptación del cuidado paliativo, el rechazo terapéutico, la readecuación terapéutica, la donación y la muerte anticipada (dentro de las condiciones de despenalización del homicidio por piedad).

La voluntad anticipada no es un sinónimo de eutanasia, como tampoco lo es el derecho a una muerte digna, como quiera que la voluntad anticipada signifique prever en el presente una decisión que, ante circunstancias determinadas frente a la vida, se adoptaría por parte del titular del derecho, y su mandato es que así se cumpla. El derecho a morir dignamente es evitar el sufrimiento a una persona que así lo solicita cuando se halla en el estadio terminal de una enfermedad grave e incurable proporcionándole la mitigación del dolor y garantizando que no será sujeto de tratamientos infructuosos, y la eutanasia es permitir la llegada del final de la vida ante las mismas circunstancias: de tal manera que la eutanasia es una de las formas para morir dignamente.
El derecho a la suscripción de voluntad anticipada se les reconoce a las personas; no obstante, pese a los antecedentes jurídicos que reconocen en Colombia el derecho de los menores a participar en la toma de decisiones de salud que les conciernen (especialmente cuando estos afectan de manera definitiva su vida, su salud y su integridad), a dicho grupo etario, al no ser capaz, se le ha negado el ejercicio de ese derecho. No obstante, consideramos que el menor de edad no puede sustraerse de tal condición; por tal motivo, debe permitírsele la posibilidad no solo de decidir, sino también de dejar plasmada dicha decisión, la cual deberá ser observada en un ámbito de protección de los derechos del menor. Por otra parte, la posibilidad de plasmar dicho documento permite que se haga a través de cualquier medio documental legalmente reconocido.

No hay claridad sobre las circunstancias en las cuales es nula o se debe revocar la voluntad anticipada, como sí es explícita en otras regulaciones.

Finalmente, las siguiente son problemáticas relacionadas con su adecuada implementación en Colombia: la escasa difusión en la población general del derecho a suscribir voluntades anticipadas, la escasa formación de los profesionales de la salud sobre el tema y la ausencia de registros nacionales respaldados por el Estado que faciliten el acceso de los profesionales de la salud a sistemas de información en los cuales reposen la totalidad de las voluntades anticipadas. 


\section{Referencias}

Argentina, Senado y Cámara de Diputados de la Nación Argentina (2009). "Ley 26529". Derechos del paciente en su relación con los profesionales e instituciones de la salud. Buenos Aires, Argentina. Recuperado de http://www.infoleg.gov.ar/infolegInternet/anexos/160000-164999/160432/ norma.htm.

Ariza, A. R., Garza, O. M., Guzmán, D. C. C., Escamilla, C. M. S. et al. (2008). La voluntad anticipada. Un dilema ético sustentado en una Ley vigente. Medicina Interna de México, 24(5), 353-356.

Asociación Médica Mundial (AMM) (1998). Declaración de Ottawa de la AMM sobre el derecho del niño a la atención médica. Recuperado de http://www.unav.es/cdb/ ammottawa3.html.

Beauchamp, T. L. y Childress, J. F. (2012). Principles of biomedical ethics (6ta. edición). Nueva York: Oxford University Press.

Cantú-Quintanilla, G., Ubiarco, V., Medeiros-Domingo, M., Gracida-Juárez, C. etal. (2012). The federal district law on advance directives in Mexico: transplants and ideology. Persona y Bioética, 16(1), 11-17.

Cañas, G. (17 de marzo de 2015). Francia aprueba el derecho de todos los pacientes a la sedación terminal. El País Internacional. Recuperado de http://internacional. elpais.com/internacional/2015/03/17/ actualidad/1426611595_636064.html.

Castiñeira, Á. y Lozano, J. (2002). ¿Qué puede aportar el debate ético contemporáneo a los científicos? España: Proa.

Colombia, Congreso Nacional. (27 de febrero de 1981). "Ley 23 de 1981, por la cual se dictan normas en materia de ética médica”. Diario Oficial, 35.711. Bogotá, Colombia.

Colombia, Congreso Nacional. (8 de marzo de 2006). "Ley 1098 de 2006, por la cual se expide el código de infancia y adolescencia”. Diario Oficial 46446. Bogotá, Colombia.

Colombia, Congreso Nacional. (2014). "Ley 1733 de 2014, Ley Consuelo Devis Saavedra, mediante la cual se regulan los servicios de cuidados paliativos para el manejo integral de pacientes con enfermedades terminales, crónicas, degenerativas e irreversibles en cualquier fase de la enfermedad de alto impacto en la calidad de vida". Bogotá, Colombia. Recuperado de http://wsp.presidencia.gov.co/ Normativa/Leyes/Documents/LEY\%20 $1733 \% 20$ DEL \% 2008\%20DE\% 20 SEPTIEMBRE\%20DE\%202014.pdf.

Colombia, Ministerio de Salud. (1991). "Resolución 13437 de 1991, por la cual se constituyen los comités de ética hospitalaria y se adoptan el decálogo de los derechos de los pacientes". Bogotá, Colombia. Recuperado de http:// www.compensar.com/doc/sal/Resolucion_\%2013437_\%201991.pdf.

Colombia, Ministerio de Salud. (2015). "Resolución 1216 de 2015, por medio de la cual se da cumplimiento a la orden cuarta de la sentencia T-970 de 2014 de la Honorable Corte Constitucional en relación con las directrices para la organización y funcionamiento de los Comités para hacer efectivo el derecho a morir con dignidad". Bogotá, Colombia. Recuperado de http:// www.dmd.org.co/pdf/Eutanasia_resolucion-1216-de-2015.pdf. 
Colombia, Presidencia de la República. (5 de agosto de 2004). "Decreto 2493 de 2004, por el cual se reglamentan parcialmente las Leyes 9a de 1979 y 73 de 1988, en relación con los componentes anatómicos". Diario Oficial 45631. Bogotá, Colombia.

Consejo de Europa. (1976a). "Resolución 613, sobre los derechos de los enfermos y moribundos". Asamblea Parlamentaria del Consejo de Europa, adoptada el 29 de enero de 1976. Recuperado de http:// www.unav.es/cdb/coeconvencion.html.

Consejo de Europa. (1976b). "Recomendación 779, sobre los derechos de los enfermos y moribundos". Asamblea Parlamentaria del Consejo de Europa, adoptada el 29 de enero de 1976. Recuperado de http://www.observatoriodepaliativos.org/legislacion-europea.

Consejo de Europa. (1997). "Convenio relativo a los derechos humanos y la biomedicina". Recuperado de http:// www.unav.es/cdb/coeconvencion.html.

Consejo de Europa. (1999). "Recomendación 1418, sobre protección de los derechos humanos y la dignidad de los enfermos terminales y moribundos". Recuperado de http://www.unav.es/cdb/ acoerec99-1418.html.

Duque, J. H. (2014). Declaraciones de voluntad anticipada: la atención en situaciones críticas del futuro. El Pulso, 16(196). Recuperado de http://www. periodicoelpulso.com/html/150lene/ general/general-08.htm.

España, Jefatura de Estado (15 de noviembre de 2002). "Ley 41, básica reguladora de la autonomía del paciente y de derechos y obligaciones en materia de información y documentación clínica”. BOE 274. Madrid: España.

España, Ministerio de Sanidad y Consumo (2007). "Real Decreto 124, por el que se regula el Registro nacional de instrucciones previas y el correspondiente fichero automatizado de datos de carácter personal". BOE del 15 de febrero. Madrid, España.

Fundación Pro Derecho a Morir Dignamente (DMD). (s. f.). Documento "Esta es mi voluntad". Recuperado de http://www. dmd.org.co/pdf/mivoluntad.pdf.

Howard Zuluaga, M. A. (2012). Las declaraciones de voluntad anticipada y la autonomía de la persona. Revista de Derecho de la Universidad de Montevideo, 11(21), 173-197.

Jiménez Heranz, M. D. (2009). Instrucciones previas el derecho a la autodeterminación del paciente terminal. En F. Bandres Moya y S. Delgado Bueno (Eds.), Biomedicina y derecho sanitario (p. 470). Madrid: Ademas Comunicaciones.

León Correa, F. J. (2008). Las voluntades anticipadas: análisis desde las experiencias en ética clínica. Revista Colombiana de Bioética, 3(2), 83-101.

Leone, S. y Privitera, S. (1994). Dizionario de bioetica. Bologna: Edizioni Dehoniane.

Martínez, J. A. (2011). La negativa al tratamiento y los actos de voluntad anticipada. Posibilidades para su regulación en el ordenamiento jurídico cubano. Bioética, 11(3), 9-17.

México, Comisión Nacional de Bioética. (s. f.). Voluntades anticipadas. Reflexiones bioéticas sobre el final de la vida. Recuperado de http://conbioetica-mexico. 
salud.gob.mx/descargas/pdf/voluntades_anticipadas.pdf.

Ortiz Rubio, J. (2009). Consentimiento informado en la especialidad de psiquiatría. Bioética, 18-21. Recuperado de http://www.cbioetica.org/revista/93/931821.pdf.

Tobar Torres, J. A. (2012). Las directivas anticipadas, la planificación anticipada de la atención y los derechos a la dignidad y autonomía del paciente. Estado de la cuestión a nivel internacional y su posibilidad de ejercicio en el derecho colombiano. Revista Colombiana de Bioética, 7(1), 140-162.

Turcott Cárdenas, A. (s. f.). La causa interpretación comparativa del derecho francés y español con el derecho mexicano en su origen. Recuperado de http://www. juridicas.unam.mx/publica/librev/rev/ facdermx/cont/239/art/art11.pdf.
Unicef. (1989). Convención sobre los derechos del niño. Recuperado de https://www. unicef.es/sites/www.unicef.es/files/ CDN_06.pdf.

Uruguay, Representantes de la República Oriental de Uruguay (2009). "Ley 18473". Voluntad anticipada. Montevideo. Recuperado de http://www.parlamento.gub.uy/leyes/AccesoTextoLey. asp?Ley $=18473 \&$ Anchor $=$

Vieira Bomtempo, T. (2012). Diretivas antecipadas: instrumento que assegura a vontade de morrer dignamente. Revista de Bioética y Derecho, (26), 22-30.

Yllera Sanz, C. (2014). ¿Cumplimos nuestros deberes respecto a los documentos de voluntades anticipadas? Revista de Bioética y Derecho, (32), 82-94.

Zappalá, F. (2008). Declaraciones anticipadas de tratamiento médico o mal denominado testamento biológico. Criterio Jurídico, 8(1), 243-268. 